\title{
Basic fibroblast growth factor promotes macaque follicle development in vitro
}

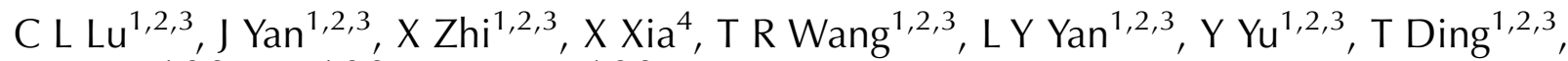 \\ J M Gao ${ }^{1,2,3}, \mathrm{R} \mathrm{Li}^{1,2,3}$ and J Qiao ${ }^{1,2,3}$
}

${ }^{1}$ Department of Obstetrics and Gynecology, Medical Center for Human Reproduction, Peking University Third Hospital, Beijing 100191, China, ${ }^{2}$ Key Laboratory of Assisted Reproduction, Ministry of Education, Beijing, China, ${ }^{3}$ Beijing Key Laboratory of Reproductive Endocrinology and Assisted Reproduction, Beijing, China and ${ }^{4}$ Center for Reproductive Medicine, Peking University Shenzhen Hospital, No. 1120 Lotus Road, FuTian District, Shenzhen, Guangdong 518000, China

Correspondence should be addressed to J Qiao; Email: jie.qiao@263.net

\begin{abstract}
Fertility preservation is an important type of frontier scientific research in the field of reproductive health. The culture of ovarian cortices to i) initiate primordial follicle growth and ii) procure developing follicles for later oocyte maturation is a promising fertility preservation strategy, especially for older women or cancer patients. At present, this goal remains largely unsubstantiated in primates because of the difficulty in attaining relatively large follicles via ovarian cortex culture. To overcome this hurdle, we cultured macaque monkey ovarian cortices with FSH, kit ligand (KL), basic fibroblast growth factor (bFGF), and/or epidermal growth factor (EGF). The various factors and factor combinations promoted primordial follicle development to different extents. Notably, both bFF (bFGF, $100 \mathrm{ng} / \mathrm{ml}$ and FSH, $50 \mathrm{ng} / \mathrm{ml})$ and $\mathrm{KF}(\mathrm{KL}, 100 \mathrm{ng} / \mathrm{ml}$ and $\mathrm{FSH}, 50 \mathrm{ng} / \mathrm{ml})$ contributed to the activation of primordial follicles at day 12 (D12) of culture, whereas at D18, the proportions of developing follicles were significantly higher in the bFF and KF groups relative to the other treatment groups, particularly in the bFF group. Estradiol and progesterone production were also highest in the bFF group, and primary follicle diameters were the largest. Up until D24, the bFF group still exhibited the highest proportion of developing follicles. In conclusion, the bFGF-FSH combination promotes nonhuman primate primordial follicle development in vitro, with the optimal experimental window within 18 days. These results provide evidence for the future success of human ovarian cortex culture and the eventual acquisition of mature human follicles or oocytes for fertility restoration.
\end{abstract}

Reproduction (2015) 149 425-433

\section{Introduction}

Fertility preservation is a critical frontier research area in the field of reproductive health. Significant advances have recently been made in fertility management, especially for older female subjects who desire later fertility and for young cancer patients. In the latter case, cryopreservation of ovarian tissues before chemoradiotherapy or treatment of estrogen-sensitive tumors, followed by orthotopic or heterotopic implantation after symptom remission, can restore the ovaries and protect reproductive function. Worldwide, more than 30 live births have been reported in cancer patients after transplantation of frozen/thawed ovarian tissues (Gamzatova et al. 2014). However, in instances of leukemia or other cancers, grafting is associated with a risk of disease recurrence because reintroduction of malignant cells into the cryopreserved ovarian tissue can take place in patients with leukemia (Donnez et al. 2006, Dolmans et al. 2010, Rosendahl et al. 2010). Therefore, to avoid the transfer of malignant cells, ovarian cortex culture ensuing in vitro differentiation of ovarian follicles or immature cumulus-oocyte complexes into mature oocytes is the best and perhaps only realistic option for fertility preservation (Picton et al. 2008).

We and numerous other research groups have attempted to culture isolated, preantral follicles for several days, with some preliminary success (Telfer et al. 2008, Amorim et al. 2009, Xu et al. 2009, 2013, Desai et al. 2010). Nonetheless, preantral follicles and oocytes are scarce, and preantral follicles can only rarely be isolated in clinical settings, particularly where estrogen-sensitive cancer patients and older subjects are concerned. Thus, ovarian cortex culture and primordial follicle activation are especially necessary to meet the demand for fertility preservation.

Initial attempts to promote primordial follicle development in vitro focused on the growth of isolated follicles. Many investigators clearly showed that it is fairly 
straightforward to harvest rodent primordial follicles by mechanical means, while in the tougher ovarian cortex of large animals and human beings, primordial follicles must be isolated via mechanical together with enzymatic methods (Greenwald \& Moor 1989, Oktay et al. 1997, Abir et al. 1999, Rice et al. 2008). However, the lack of influence from extrafollicular cortical cells leads to the demise of isolated primordial follicles in vitro. Therefore, in large animals and humans, the in vitro growth and maturation of primordial oocytes are only likely to be initiated in situ by culture of the ovarian cortex. Preantral follicles can then be isolated and grown into the antral stage of folliculogenesis. Accordingly, ovarian cortex culture and primordial follicle activation are both technically necessary for primordial follicle growth and development.

Activation of primordial follicles is modulated through an intricate balance between stimulatory and inhibitory factors (Picton 2001, Picton et al. 2008). Hsueh AJ. Laboratory has recently demonstrated fragmentation of ovary-disrupted ovarian Hippo signaling, leading to increased expression of downstream growth factors and promotion of follicle growth. Phosphatidylinositol 3 kinase (PI3K)/AKT pathway allows short-term activation of dormant ovarian follicles in vitro ( $\mathrm{Li}$ et al. 2010, Kawamura et al. 2013). Addition of FSH, epidermal growth factor (EGF), and basic fibroblast growth factor (bFGF) are beneficial for the follicular transition (Matos et al. 2007a,b, Peng et al. 2010). Kit ligand (KL)/stem cell factor was one of the first identified factors in the promotion of primordial follicle development (Parrott \& Skinner 1999); importantly, KL also promotes follicle survival and antral cavity formation of caprine preantral follicles in vitro in the absence of FSH (Lima et al. 2011).

EGF, for its part, is essential for the maintenance of intraovarian primordial follicle viability in the prepubertal cat, albeit without promoting follicle activation (Fujihara et al. 2014). Furthermore, EGF and FSH both increase the size of goat primordial follicles by stimulating oocyte growth (Silva et al. 2004). Contrarily, bFGF initiates the activation of primordial follicles in the rat (Kezele et al. 2002), and interactions between bFGF and $\mathrm{KL}$ effectively induce the primordial-to-primary follicle transition in the rat ovary (Nilsson \& Skinner 2004). Matos et al. $(2007 a, b, c)$ showed that bFGF at a concentration of $50 \mathrm{ng} / \mathrm{ml}$ not only maintained the morphological integrity of caprine preantral follicles in culture for 5 days, but also stimulated the activation of primordial follicles and the subsequent growth of the activated follicles. FSH at $50 \mathrm{ng} / \mathrm{ml}$ functioned in a manner reminiscent of bFGF, as did the combination of FSH and bFGF (i.e., by stimulating the activation as well as the growth of primordial follicles into mature follicles) (Matos et al. 2007a,b,c). Other investigators similarly found that after 7-day culture of the goat ovarian cortex, FGF-10 upheld preantral follicle morphological integrity and promoted activated follicle growth in vitro (Chaves et al. 2010). In addition, high doses of bFGF reportedly enhanced follicle development in frozen/thawed ovarian tissues of cancer patients under serum-free conditions, and also in cultured human fetal ovarian tissue (Garor et al. 2009).

Nevertheless, few reports are available regarding the impact of growth factors and hormone signals on human or nonhuman primate ovarian tissue culture, and the coordination of various growth factors and hormones required for proper follicle development and oocyte maturation in vitro remains challenging. Therefore, this study set out to evaluate the effects of EGF, bFGF, and KL in combination with FSH on the in vitro development of primordial follicles derived from the ovarian cortex of the macaque monkey.

\section{Materials and methods}

\section{Ethical approval}

This study was approved by the Institutional Review Board of Pecking University Third Hospital (Beijing, China). Acquirement of the macaque ovaries was in accordance with the Guide for Care and Use of Laboratory Animals of Peking University (Beijing, China). The protocol was approved by the Institutional Review Board of Animal Experiments of Peking University Third Hospital.

\section{In vitro culture}

Immediately after slaughter, ovaries were collected by laparoscopy from 2- to 3-year-old female macaque monkeys with normal body condition. The ovaries were transported in Leibovitz's L-15 Medium (Invitrogen) supplemented with $1 \%$ human serum albumin (LifeGlobal, Guilford, CT, USA), $100 \mathrm{IU} / \mathrm{ml}$ penicillin, and $100 \mu \mathrm{g} / \mathrm{ml}$ streptomycin (Invitrogen), and placed on ice for transport to the laboratory within $2 \mathrm{~h}$. The ovaries were washed three times with $0.9 \%$ saline supplemented with penicillin and streptomycin. Next, in each replicate, the cortices of eight ovaries were dissected into cortical plate fragments $(\sim 3 \mathrm{~mm}$ long $\times 3 \mathrm{~mm}$ wide $\times 1 \mathrm{~mm}$ thick), and 100 fragments were selected for the experiments described below. Four fragments were histologically analyzed as uncultured controls, and the remaining 96 fragments were randomly divided into the wells of a 24-well plate, with a total of six different treatments (four fragments per well, yielding a total of 16 fragments per treatment).

Each well contained $250 \mu \mathrm{l}$ of culture media, consisting of basic medium (alpha-minimum essential medium ( $\alpha$-MEM) plus $1 \%$ human serum albumin, $1 \%$ insulin/transferrin/ selenium $(5 \mu \mathrm{g} / \mathrm{ml}$ insulin, $5 \mu \mathrm{g} / \mathrm{ml}$ transferrin, and $5 \mathrm{ng} / \mathrm{ml}$ selenium, ITS) (Invitrogen), $50 \mathrm{mg} / \mathrm{ml} 6 \%$ ascorbic acid (Sigma-Aldrich), $2 \mathrm{mM}$ L-glutamine, $75 \mathrm{mg} / \mathrm{ml}$ penicillin $\mathrm{G}$, and $50 \mathrm{mg} / \mathrm{ml}$ streptomycin. In some cases, the medium was further supplemented with $50 \mathrm{ng} / \mathrm{ml} \mathrm{FSH}$ or FSH plus EGF $(75 \mathrm{ng} / \mathrm{ml})$, bFGF $(100 \mathrm{ng} / \mathrm{ml})$, and/or $\mathrm{KL}(100 \mathrm{ng} / \mathrm{ml})$. The concentrations of the growth factors were based on their stimulatory effects in earlier studies (Muruvi et al. 2005, Matos et al. 2007a,b, Peng et al. 2010). The six treatment groups 
finally employed in this study were as follows: i) $\alpha$-MEM, ii) $\mathrm{FSH}$, iii) bFF (bFGF and FSH), iv) EF (EGF and FSH), v) KF ( $\mathrm{KL}$ and $\mathrm{FSH}$ ), and vi) EbKF (EGF, bFGF, KL, and FSH). The medium was changed every 3 days. Noncultured fragments were fixed in $10 \%$ formaldehyde on day 0 (D0), while cultured fragments were fixed in $10 \%$ formaldehyde on D6, D12, D18, and D24 post-culture for histological analysis.

\section{Morphological classification and histological analysis of growing follicles}

The fixed samples were embedded in paraffin wax, and every 12th serial section (5- $\mu$ m-thick) was chosen to prevent follicles from being counted twice. Routine hematoxylin and eosin staining was performed for histologic examination under light microscopy. Only normal or healthy follicles (spherical shape, oocyte-bearing, intact theca, regularly distributed granulosa cells) with visible oocyte nuclei were recorded.

Follicular stages were classified into five types based on the descriptions of Fortune (2003), as follows: i) primordial follicles (containing an oocyte surrounded by a single layer of flattened granulosa cells); ii) primary follicles (containing an oocyte surrounded by a single layer of cuboidal granulosa cells); iii) secondary follicles (containing a growing oocyte surrounded by two to five layers of granulosa cells); iv) preantral follicles (containing six or more layers of granulosa cells, or with a diameter of $>120 \mu \mathrm{m}$, and without any signs of antrum formation); and v) antral follicles (containing an oocyte surrounded by multiple layers of granulosa cells, and with an antrum). In this study, follicles from groups 2, 3, 4, and 5 were collectively termed developing follicles (Gougeon 1996, Oktay et al. 1998, Fortune 2003). All follicles showing no signs of atresia were counted for each treatment group at D6, D12, D18, and D24. Follicle diameters were measured as the mean of two perpendicular diameters. The results of independent triplicate experiments were presented after Statistical analyses (see below).

\section{Hormone assay}

Culture media from all treatment groups at each time point (D6, D12, D18, and D24) were collected and stored at $-80{ }^{\circ} \mathrm{C}$ until use for the hormone assay. The levels of $17 \beta$-estradiol $\left(E_{2}\right)$ were measured by using a commercial RIA kit at a commercial laboratory (Chemclin Biotech Co. Ltd, Beijing, China). The sensitivity of the $E_{2}$ assay was $<3 \mathrm{pg} / \mathrm{ml}$. Progesterone levels were assessed by using an ELIZA kit (Roche Diagnostics $\mathrm{GmbH}$ ). The sensitivity of the progesterone assay was $0.03 \mathrm{ng} / \mathrm{ml}$.

\section{Statistical analyses}

The percentages of follicles at different developmental stages and the follicle diameters were calculated and analyzed by first assessing the normality and homogeneity of the data variance, and then by conducting a two-way ANOVA. Next, a one-way ANOVA was conducted, followed by appropriate post hoc analyses with Tukey's test and the least square difference test. Hormone production levels were analyzed by conducting a repeated measures ANOVA. The values were considered to be

Table 1 Numbers of surviving primordial, primary, secondary, and preantral, and antral follicles in the ovarian cortex on D0, D6, D12, D18, and D24 post-culture.

\begin{tabular}{|c|c|c|c|c|c|c|}
\hline & \multirow[b]{2}{*}{ Days } & \multicolumn{5}{|c|}{ Surviving follicles $(n)$} \\
\hline & & Primordial & Primary & Secondary & Pre-/antral & Total \\
\hline Uncultured & 0 & 890 & 33 & 39 & 22 & 984 \\
\hline \multirow[t]{4}{*}{$\alpha-M E M$} & 6 & 510 & 52 & 30 & 5 & 597 \\
\hline & 12 & 150 & 81 & 34 & 13 & 278 \\
\hline & 18 & 35 & 20 & 7 & 0 & 62 \\
\hline & 24 & 47 & 14 & 7 & 0 & 68 \\
\hline \multirow{4}{*}{ FSH } & 6 & 154 & 12 & 12 & 3 & 181 \\
\hline & 12 & 237 & 165 & 43 & 3 & 448 \\
\hline & 18 & 240 & 72 & 23 & 5 & 340 \\
\hline & 24 & 288 & 88 & 9 & 2 & 387 \\
\hline \multirow[t]{4}{*}{ bFF } & 6 & 414 & 45 & 23 & 9 & 491 \\
\hline & 12 & 121 & 72 & 12 & 11 & 216 \\
\hline & 18 & 307 & 144 & 56 & 13 & 520 \\
\hline & 24 & 87 & 54 & 11 & 0 & 152 \\
\hline \multirow[t]{4}{*}{$\mathrm{EF}$} & 6 & 1022 & 101 & 74 & 20 & 1217 \\
\hline & 12 & 77 & 55 & 41 & 11 & 184 \\
\hline & 18 & 259 & 36 & 16 & 3 & 314 \\
\hline & 24 & 145 & 65 & 6 & 0 & 216 \\
\hline \multirow[t]{4}{*}{ KF } & 6 & 253 & 67 & 31 & 16 & 367 \\
\hline & 12 & 196 & 172 & 125 & 11 & 504 \\
\hline & 18 & 185 & 50 & 1 & 0 & 236 \\
\hline & 24 & 38 & 12 & 4 & 1 & 55 \\
\hline \multirow{4}{*}{ EbKF } & 6 & 417 & 81 & 12 & 3 & 513 \\
\hline & 12 & 152 & 61 & 72 & 10 & 295 \\
\hline & 18 & 203 & 36 & 18 & 5 & 262 \\
\hline & 24 & 104 & 33 & 30 & 0 & 167 \\
\hline
\end{tabular}

The six experimental groups were as follows: i) $\alpha$-MEM, ii) FSH, iii) bFF (bFGF and FSH), iv) EF (EGF and FSH), v) KF (KL and FSH), and vi) EbKF (EGF, bFGF, KL, and FSH). $\alpha$-MEM, $\alpha$-minimal essential medium; bFGF, basic fibroblast growth factor; EGF, epidermal growth factor; KL, kit ligand. 


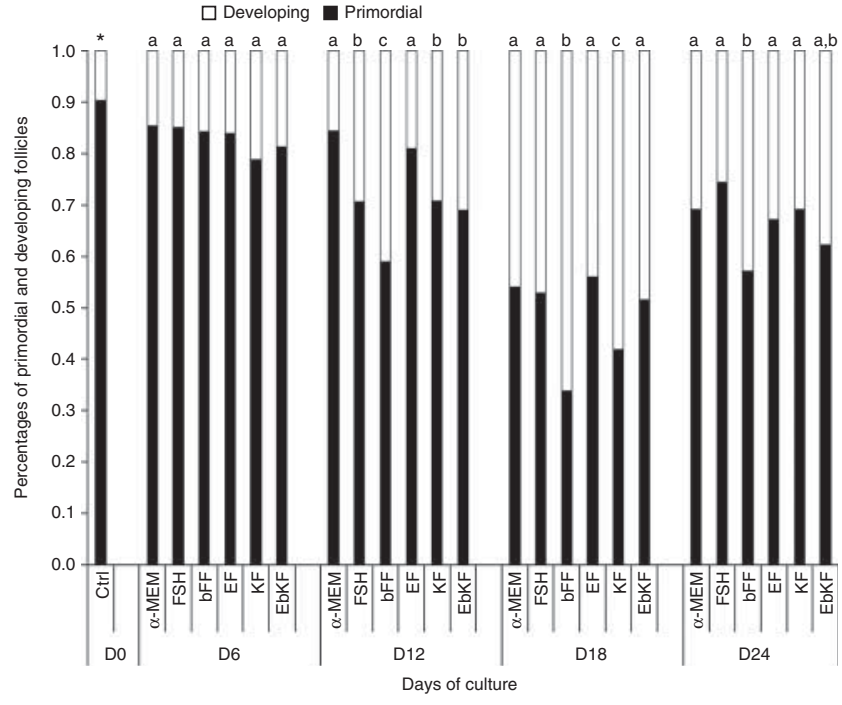

Figure 1 Fractions of primordial and developing follicles in the six treatment groups ( $\alpha-M E M, F S H, b F F(b F G F$ and FSH), EF (EGF and FSH), $\mathrm{KF}(\mathrm{KL}$ and $\mathrm{FSH})$, and EbKF (EGF, bFGF, KL, and FSH)) at selected time points (D6, D12, D18, and D24). The uncultured control group is denoted by D0. The asterisk $(*)$ indicates that the cultured treatment groups were significantly different from the uncultured control (Ctrl) group (D0) $(P<0.05)$. Different lowercase letters $(\mathrm{a}, \mathrm{b}$, and c) indicate significant differences $(P<0.05$ or $P<0.01)$ between the various treatments on the same day of culture.

statistically significant at $P<0.05$. SPSS19 Software for Windows (SPSS Science) was used for all calculations.

\section{Results}

\section{Development of follicles in the in vitro-cultured ovarian cortex}

The numbers of surviving primordial, primary, secondary, preantral, and antral follicles were counted for the uncultured control at D0 and the various treatment conditions at D6, D12, D18, and D24 (Table 1). We then used these numbers to calculate the fractions of primordial and developing follicles at each stage of development.

Figure 1 shows the effects of $\alpha$-MEM, FSH, bFF, EF, KF, and EbKF on follicle maturation at D6, D12, D18, and D24. In general, the fraction of primordial follicles in the ovarian cortex sections decreased with each time points of culture, while the fraction of developing follicles increased. At 6 days in culture (D6), the fractions of primordial follicles $(\sim 0.80-0.85)$ and developing follicles $(\sim 0.15-0.20)$ were similar across treatment groups, whereas at D12, the developing follicles increased in number, with the highest fraction observed in the bFF group. After 18 days in culture (D18), the fraction of developing follicles showed significant increases in both the bFF and KF groups relative to the other groups, but was still highest in the bFF group. Up until D24, bFF treatment continued to enhance follicle development compared with the other five groups, although all groups showed attenuated follicle development at D24 vs D18. The addition of the factor EbKF (the combination of bFGF, EGF, KL, and FSH) did not significantly increase the percentage of developing follicles at any time point compared with single factors alone (Fig. 1).

Figure 2 shows the time-dependent actions of $\alpha$-MEM, $\mathrm{FSH}, \mathrm{bFF}, \mathrm{EF}, \mathrm{KF}$, and EbKF on follicle development. The numbers of developing follicles increased gradually with time in culture from D6 to D18 for all treatment groups, with the highest numbers observed at D18, along with the lowest numbers of primordial follicles. However, in all cases, the percentage of developing follicles decreased at D24 to the levels similar to those observed at D12.

\section{Histological observation of the in vitro-cultured ovarian cortex}

Figure 3 shows representative histological hematoxylin and eosin-stained images of the ovarian cortex in the $\alpha-M E M$ and bFF groups on D6, D12, and D1 8 of culture. Every stage of follicle development is illustrated. Normal (healthy) follicles were spherical in shape and contained oocytes surrounded by regular granulosa cells and a circular distribution of theca cells. On the other hand, nearly only primordial follicles were observed in the uncultured control group (i.e., the untreated ovarian cortex at D0). The majority of the follicles in the $\alpha$-MEM group remained in the primordial stage at D6, with only

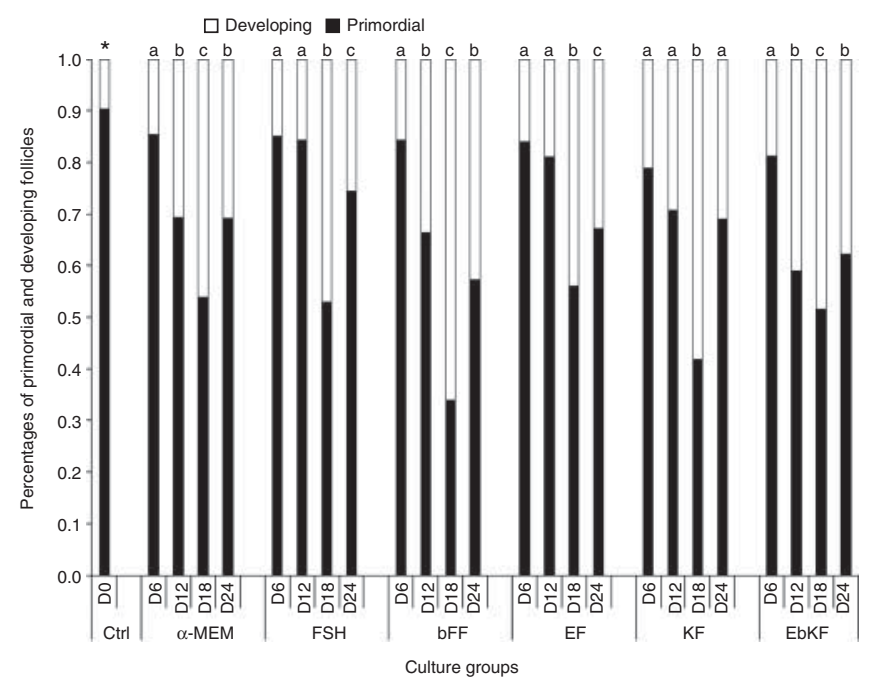

Figure 2 Time-dependent effects of $\alpha-M E M, F S H, b F F, E F, K F$, and EbKF on follicle development at D6, D12, D18, and D24. The uncultured control group is denoted by D0. The asterisk $\left({ }^{*}\right)$ indicates that the cultured treatment groups were significantly different from the uncultured control (Ctrl) group. Different lowercase letters (a, b, and c) indicate significant differences between the same treatment on different days of culture $(P<0.05$ or $P<0.01)$. 


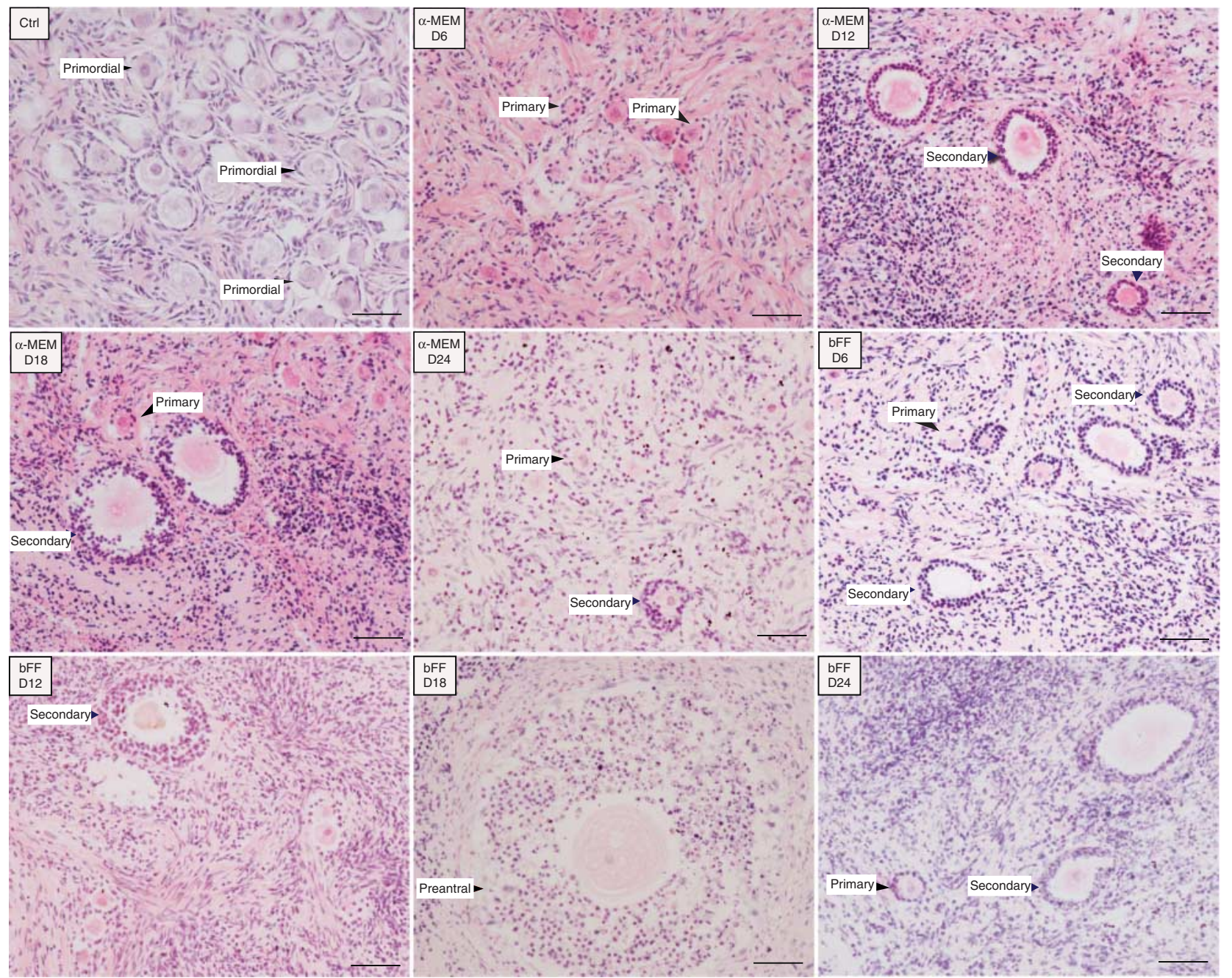

Figure 3 Histological examination of the ovarian cortex. Images are shown at $20 \times$ magnification for the uncultured control group (Ctrl), the cultured $\alpha$-MEM group at D6, D12, D18, and D24, and the cultured bFF group at D6, D12, D18, and D24. Different types of follicles were labeled with the following: primordial, primary, secondary, and preantral. Scale bar: $50 \mu \mathrm{m}$.

a few primary follicles discerned. At D12, some of the primordial follicles in the $\alpha$-MEM group became activated, and developed to the primary or preantral stage. However, developing follicles no longer grow significantly at D18. Contrarily, an obvious tendency toward follicle development was observed in the bFF group, especially from D6 to D18. All groups exhibited decreased numbers of viable follicles and/or increased numbers of degenerated follicles until D24 compared with D18. Degenerated follicles corresponded to follicles with a retracted oocyte or a pyknotic nucleus, and/or those surrounded by disorganized granulosa cells that were detached from the basement membrane (Matos et al. 2007a,b).

\section{Assessment of hormone production}

We next assessed hormone production in the various treatment groups (Fig. 4). In general, all six treatments increased progesterone production over time in culture, with the highest overall progesterone levels seen at D18. In detail, progesterone levels increased from D6 to D12, with the most progesterone production at D12 in the bFF and KF groups (bFF vs KF group, $50.6 \mathrm{ng} / \mathrm{ml}$ vs $48.6 \mathrm{ng} / \mathrm{ml}$ ). At D18, progesterone production in the bFF group rose to $233.9 \mathrm{ng} / \mathrm{ml}$; this value was significantly higher than that observed in any of the other treatment groups at the same time point. However, progesterone levels markedly decreased from D18 to D24 in the bFF group, and likewise in the FSH, EF, and EbKF groups. Progesterone production remained fairly constant in the $\alpha-M E M$ and KF groups during this time period (Fig. 4A and B).

Similar results were observed for $E_{2}$ production. The $E_{2}$ level in the bFF group was relatively high at D12, and significantly higher than in any of the other treatment groups at D18. $E_{2}$ levels were also higher at D18 in the $\mathrm{FSH}$ and KF groups vs the EF and EbKF groups. At D24, 

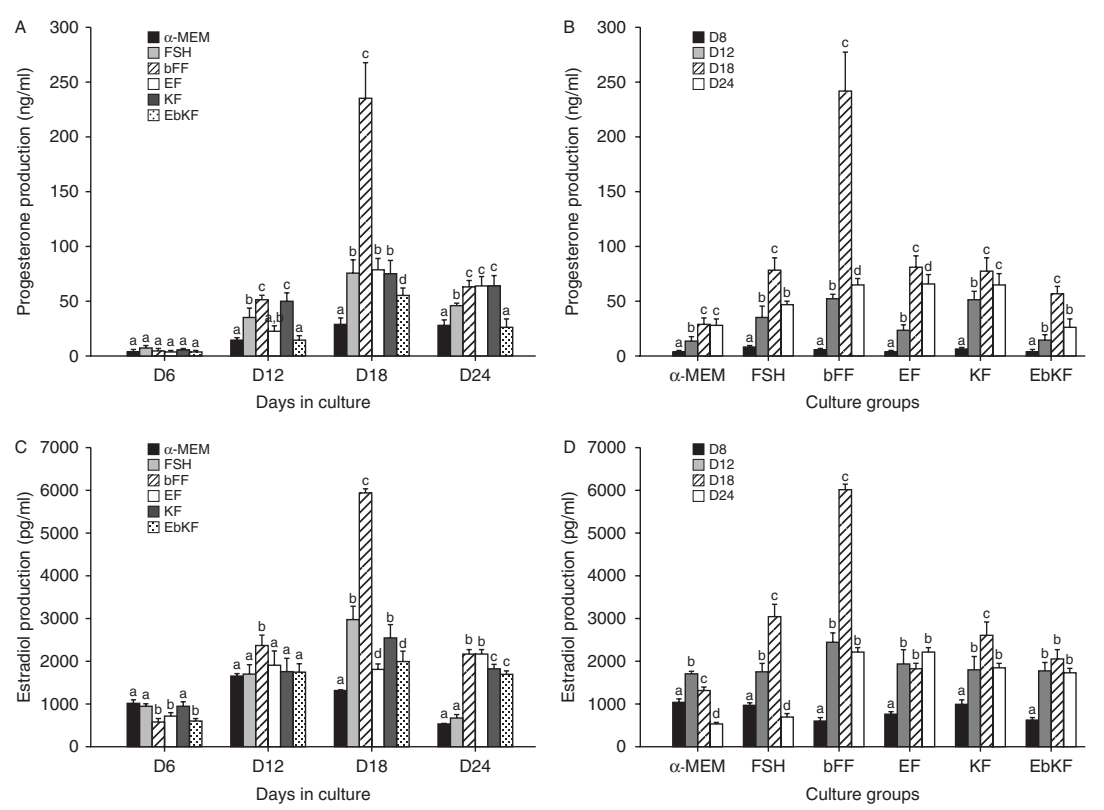

Figure 4 Hormone profiles of progesterone (A and $\mathrm{B})$ and estradiol ( $\mathrm{C}$ and $\mathrm{D})$ in the six treatment groups ( $\alpha-M E M, F S H, b F F, E F, K F$, and EbKF) at D6, $\mathrm{D} 12, \mathrm{D} 18$, and D24. Data are given as the means \pm the S.E.M. Different lowercase letters $(a, b$, and c) indicate significant differences between the various treatments on the same day of culture (A and $\mathrm{C}$ ) or significant differences between the same treatment on different days of culture $(\mathrm{B}$ and $\mathrm{D})(P<0.05)$.

the $E_{2}$ levels generally decreased to lower levels, which were similar to those observed at D12, except for in the $\alpha-M E M$ and FSH groups. In these two groups, $E_{2}$ levels were significantly lower at D24 than at D12 (Fig. 4C and D).

\section{Measurement of follicle diameters}

Lastly, the diameters of all surviving primordial, primary, and secondary follicles were measured at D6, D12, D18, and D24 (Fig. 5). No significant differences were observed for primordial follicle diameters within each treatment group across the various time points in culture. However, the diameters of the primordial follicles were significantly larger in the bFF group than in the $\alpha$-MEM, $\mathrm{FSH}$, and KF groups $(P=0.004,0.038$, and 0.013 respectively; not labeled in the figure). For the primary follicles, significant differences were generally seen between the D6, D12, D18, and D24 time points, in that the diameters at D24 were significantly smaller than those at D6, D12, and D18 $(P=0.001,<0.001$, and 0.001 respectively; not labeled in the figure). In addition, the primary follicle diameters were clearly larger in the bFF group than in the KF and EbKF groups $(P=0.002$ and 0.003 respectively); similar results were obtained for the EF group vs the KF group $(P=0.004)$. However, no significant differences were discerned among the secondary follicle diameters.

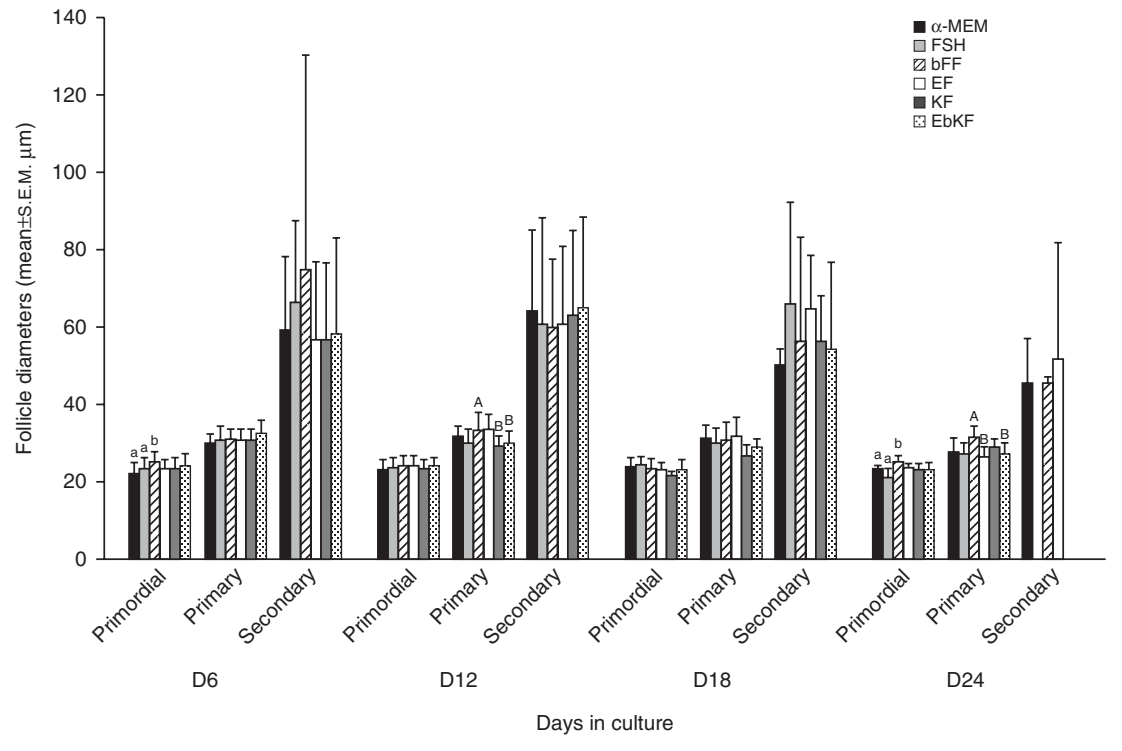

Figure 5 Effects of $\alpha$-MEM, FSH, bFF, EF, KF, and EbKF on the diameters of primordial, primary, and secondary follicles in the ovarian cortex at D6, D12, D18, and D24. Data are given as the means \pm the S.E.M. Different lowercase ( $a$ and $b$ ) and uppercase (A and B) letters indicate significant differences between the various treatment groups on the same day of culture ( $a$ and $b$ : $P<0.05 ; A$ and B: $P<0.01$ ). 


\section{Discussion}

The use of ovarian cortex culture to obtain preantral follicles is an important strategy for fertility preservation. Several factors and hormones reportedly promote the survival and activation of primordial follicles (Esmaielzadeh et al. 2013). However, until now, no efficient method was documented for early primordial follicle activation and maturation in vitro in primates. To address this shortcoming, the current study employed a macaque monkey ovarian cortex culture system to study the impact of EGF, KL, FSH, and bFGF on follicle development. The results show that $\mathrm{KL}$ and bFGF are crucial for the activation of primordial follicles. Moreover, with the extension of culture time, bFGF becomes more essential than $\mathrm{KL}$ to this process.

As noted previously, EGF critically participates in the maintenance of intraovarian primordial follicle viability in the prepubertal cat, but not in the promotion of follicle activation in cultured ovarian cortical slices. EGF exerts its actions via stimulation of MAPK and PI3K signaling pathways that, in turn, promote ovarian cell proliferation (Fujihara et al. 2014). Regardless of the presence or absence of FSH, EGF vs $\alpha$-MEM can effectively increase the daily follicular growth rate of secondary follicles during the in vitro culture of caprine preantral follicles (Celestino et al. 2011). In addition, this study demonstrated that EGF is no more efficient than FSH alone in promoting the activation of primordial follicles, given that we found no significant increase in the fraction of developing follicles in the $\mathrm{EF}$ vs $\mathrm{FSH}$ treatment group at D18 (Fig. 1). Furthermore, we failed to observe an increase in $\mathrm{E}_{2}$ and progesterone production in the $\mathrm{EF}$ (the combination of EGF and FSH) vs FSH alone treatment group at time points before D18 (Fig. 4). However, EGF apparently contributes to follicle survival in long-term cultures, because higher $\mathrm{E}_{2}$ and progesterone levels were detected in the EF vs FSH group at D24. Therefore, we conclude that although the actions of EGF and FSH are not additive in the stimulation of follicle development, EGF itself can benefit follicle survival.

$\mathrm{KL}$ produced by granulosa cells appears to stimulate oocyte growth and to initiate oocyte development in assorted animal models (Parrott \& Skinner 1999, Kezele \& Skinner 2003, Skinner 2005). For example, KL induces a primordial-to-primary follicle transition in the rat ovary (Parrott \& Skinner 1999), and likewise triggers a reduction in the percentage of primordial follicles with a concomitant increase in the percentage of primary and secondary follicles in the neonatal murine ovary (Gougeon et al. 2010). In addition, KL stimulates the activation of primordial follicles in the goat, promotes the survival of antral cavity formation by caprine preantral follicles after in vitro culture, and facilitates the growth and meiotic resumption of caprine oocytes in the absence of FSH (Celestino et al. 2010, Lima et al. 2011). However, according to another report, KL-kit interactions might not be associated with the growth initiation of primordial oocytes in the pig, although $\mathrm{KL}$ through kit does appear to enhance the survival of porcine oocytes (Moniruzzaman \& Miyano 2007).

$\mathrm{KL} /$ kit signaling through $\mathrm{PI} 3 \mathrm{~K}$ promotes ovarian follicle maturation, but is dispensable for primordial follicle activation in mice (John et al. 2009). We found that KF (the combination of $\mathrm{KL}$ and $\mathrm{FSH}$ ) increased the numbers of developing follicles in the macaque ovarian cortex, especially at D18 (Figs 1 and 2). Therefore, we propose that $\mathrm{KL}$ can activate primordial follicle development in the prepubertal macaque ovary in the presence of $\mathrm{FSH}$. Moreover, the KF combination enhanced progesterone and $E_{2}$ levels relative to $\alpha$-MEM. However, no consistently additive effects were observed for $\mathrm{KL}$ and $\mathrm{FSH}$ actions in hormone production before D18 (Fig. 4).

Studies in mammals demonstrate the expression of bFGF and its receptors in early human ovarian follicles (Yeh \& Osathanondh 1993, Quennell et al. 2004, Ben-Haroush et al. 2005). In addition, bFGF produced by the oocyte during the early stages of follicle development apparently acts on adjacent somatic cells to promote cell growth and maturation. Thus, bFGF, similar to the action of $\mathrm{KL}$, appears to be a primordial follicle-inducing factor. In support of this hypothesis, bFGF treatment dramatically decreased primordial follicle numbers and increased developing follicle numbers in cultured 4-day-old prepubertal rat ovaries, similar to the actions of KL (Nilsson \& Skinner 2004). Both bFGF and $\mathrm{KL}$ are required to optimally promote the primordial-to-primary follicle transition, and oocytederived bFGF functions in part to increase $K L$ expression in granulosa cells. Furthermore, bFGF regulates primordial follicle development to directly influence female reproductive efficiency (Nilsson et al. 2001).

Recently, we established that bFGF promotes the development of isolated preantral follicles in vitro (Wang et al. 2014). However, many infertility patients present with few or no preantral follicles, or even without any visible follicles. Ovarian cortex culture now supplies hope for fertility preservation in these follicle-deficient patients, as well as for older women with declining fertility. In this study, we found a positive role for bFF (the combination of bFGF and FSH) not only in the activation of follicle development in macaque ovarian cortex culture, but also in the growth of small-diameter follicles, including preantral follicles (Figs 1, 3, and 5). Furthermore, the hormone levels of progesterone and $E_{2}$ were remarkably higher in the bFF group compared with the other treatment groups at D18, and also reached their peak levels at D18 (Fig. 4). As such, our findings indicate that bFGF can be used in combination with FSH for in vitro follicle development during an appropriate time window spanning the primordial to preantral stage.

In this study, EbKF (the combination of EGF, bFGF, KL, and $\mathrm{FSH}$ ) was no more efficacious than bFF alone in the stimulation of follicle development (Figs 1 and 2), 
implying that EGF and $\mathrm{KL}$ cannot augment the actions of bFGF and FSH. Moreover, this study used bFGF at a concentration of $100 \mathrm{ng} / \mathrm{ml}$, although we previously employed the growth factor at $200 \mathrm{ng} / \mathrm{ml}$ for in vitro follicle culture. Whether bFGF is more effective at 100 or $200 \mathrm{ng} / \mathrm{ml}$ is unknown in future human ovarian cortex culture and will require further comparative experimentation. Regardless, bFGF almost certainly can be selected to enhance primordial follicle activation and growth to the preantral stage in humans as well as macaques.

Our research group also previously demonstrated that bFGF might substantially improve the quality of transplanted murine ovarian tissues by increasing follicle quantity and by promoting neoangiogenesis (Gao et al. 2013). At D24, the percentage of developing follicles and the levels of progesterone and $E_{2}$ were lower or similar to those at D18 and D12 (Figs 1, 2, and 4). These findings might be explained by the fact that after longterm culture of ovarian cortex, some of the follicles develop into large preantral follicles or more advanced premature follicles. These latter follicle types require more complicated culture conditions (e.g., a threedimensional culture system, which we successfully used in an earlier study of preantral follicle growth; Wang et al. 2014). We also suggest that after 18 days, the preantral follicle should be isolated from the ovarian cortex and cultured independently due to the absence of adequate nutrients and oxygen in the intact cortical tissue. In conclusion, we showed that the combination of bFGF and FSH is sufficient to activate primordial follicle development in the cultured macaque monkey ovarian cortex. The results of this study may provide a foundation for future human ovarian cortex culture to restore fertility in cancer patients and older women, and other subjects with impaired or degenerating follicles. First, our shortterm goals are to obtain preantral or antral human follicles by culturing the human ovarian cortex with bFGF and FSH, and then to isolate the follicles for further maturation in vitro; or alternatively, to attain mature human oocytes for fertility preservation or assisted reproduction.

\section{Declaration of interest}

The authors declare that there is no conflict of interest that could be perceived as prejudicing the impartiality of the research reported.

\section{Funding}

This work was supported by the National Natural Science Foundation of China (grant numbers 81300456, 81200470, and 31429004) and the National Basic Research Program of China (grant numbers 2011CB944504 and 2011CB944503).

\section{References}

Abir R, Roizman P, Fisch B, Nitke S, Okon E, Orvieto R \& Ben RZ 1999 Pilot study of isolated early human follicles cultured in collagen gels for 24 hours. Human Reproduction 14 1299-1301. (doi:10.1093/humrep/ 14.5.1299)

Amorim CA, Van Langendonckt A, David A, Dolmans MM \& Donnez J 2009 Survival of human pre-antral follicles after cryopreservation of ovarian tissue, follicular isolation and in vitro culture in a calcium alginate matrix. Human Reproduction 24 92-99. (doi:10.1093/humrep/ den343)

Ben-Haroush A, Abir R, Ao A, Jin S, Kessler-Icekson G, Feldberg D \& Fisch B 2005 Expression of basic fibroblast growth factor and its receptors in human ovarian follicles from adults and fetuses. Fertility and Sterility 84 (Suppl 2) 1257-1268. (doi:10.1016/j.fertnstert.2005.05.018)

Celestino JJ, Bruno JB, Lima-Verde IB, Matos MH, Saraiva MV, Chaves RN, Martins FS, Almeida AP, Cunha RM, Lima LF et al. 2010 Steady-state level of kit ligand mRNA in goat ovaries and the role of kit ligand in preantral follicle survival and growth in vitro. Molecular Reproduction and Development 77 231-240. (doi:10.1002/mrd.21138)

Celestino JJ, Bruno JB, Saraiva MV, Rocha RM, Brito IR, Duarte AB, Araujo VR, Silva CM, Matos MH, Campello CC et al. 2011 Steady-state level of epidermal growth factor (EGF) mRNA and effect of EGF on in vitro culture of caprine preantral follicles. Cell and Tissue Research 344 539-550. (doi:10.1007/s00441-011-1162-1)

Chaves RN, Lima-Verde IB, Celestino JJ, Duarte AB, Alves AM, Matos MH, Campello CC, Name KP, Bao SN, Buratini JJ et al. 2010 Fibroblast growth factor-10 maintains the survival and promotes the growth of cultured goat preantral follicles. Domestic Animal Endocrinology 39 249-258. (doi:10.1016/j.domaniend.2010.06.006)

Desai N, Alex A, AbdelHafez F, Calabro A, Goldfarb J, Fleischman A \& Falcone T 2010 Three-dimensional in vitro follicle growth: overview of culture models, biomaterials, design parameters and future directions. Reproductive Biology and Endocrinology 8 119. (doi:10.1186/14777827-8-119)

Dolmans MM, Marinescu C, Saussoy P, Van Langendonckt A, Amorim C \& Donnez J 2010 Reimplantation of cryopreserved ovarian tissue from patients with acute lymphoblastic leukemia is potentially unsafe. Blood 116 2908-2914. (doi:10.1182/blood-2010-01-265751)

Donnez J, Martinez-Madrid B, Jadoul P, Van Langendonckt A, Demylle D \& Dolmans MM 2006 Ovarian tissue cryopreservation and transplantation: a review. Human Reproduction Update 12 519-535. (doi:10.1093/ humupd/dml032)

Esmaielzadeh F, Hosseini SM, Nasiri Z, Hajian M, Chamani M, Gourabi H, Shahverdi AH, Vosough AD \& Nasr-Esfahani MH 2013 Kit ligand and glial-derived neurotrophic factor as alternative supplements for activation and development of ovine preantral follicles in vitro. Molecular Reproduction and Development 80 35-47. (doi:10.1002/mrd.22131)

Fortune JE 2003 The early stages of follicular development: activation of primordial follicles and growth of preantral follicles. Animal Reproduction Science 78 135-163. (doi:10.1016/S0378-4320(03)00088-5)

Fujihara M, Comizzoli P, Keefer CL, Wildt DE \& Songsasen N 2014 Epidermal growth factor (EGF) sustains in vitro primordial follicle viability by enhancing stromal cell proliferation via MAPK and PI3K pathways in the prepubertal, but not adult, cat ovary. Biology of Reproduction 90 86. (doi:10.1095/biolreprod.113.115089)

Gamzatova Z, Komlichenko E, Kostareva A, Galagudza M, Ulrikh E, Zubareva T, Sheveleva T, Nezhentseva E \& Kalinina E 2014 Autotransplantation of cryopreserved ovarian tissue - effective method of fertility preservation in cancer patients. Gynecological Endocrinology 30 (Suppl 1) 43-47. (doi:10.3109/09513590.2014.945789)

Gao JM, Yan J, Li R, Li M, Yan LY, Wang TR, Zhao HC, Zhao Y, Yu Y \& Qiao J 2013 Improvement in the quality of heterotopic allotransplanted mouse ovarian tissues with basic fibroblast growth factor and fibrin hydrogel. Human Reproduction 28 2784-2793. (doi:10.1093/humrep/det296)

Garor R, Abir R, Erman A, Felz C, Nitke S \& Fisch B 2009 Effects of basic fibroblast growth factor on in vitro development of human ovarian primordial follicles. Fertility and Sterility 91 (5 Suppl) 1967-1975. (doi:10.1016/j.fertnstert.2008.04.075)

Gougeon A 1996 Regulation of ovarian follicular development in primates: facts and hypotheses. Endocrine Reviews 17 121-155. (doi:10.1210/ edrv-17-2-121) 
Gougeon A, Delangle A, Arouche N, Stridsberg M, Gotteland JP \& Loumaye E 2010 Kit ligand and the somatostatin receptor antagonist, BIM-23627, stimulate in vitro resting follicle growth in the neonatal mouse ovary. Endocrinology 151 1299-1309. (doi:10.1210/en.20090762)

Greenwald GS \& Moor RM 1989 Isolation and preliminary characterization of pig primordial follicles. Journal of Reproduction and Fertility $\mathbf{8 7}$ 561-571. (doi:10.1530/jrf.0.0870561)

John GB, Shidler MJ, Besmer P \& Castrillon DH 2009 Kit signaling via PI3K promotes ovarian follicle maturation but is dispensable for primordial follicle activation. Developmental Biology 331 292-299. (doi:10.1016/ j.ydbio.2009.05.546)

Kawamura K, Cheng Y, Suzuki N, Deguchi M, Sato Y, Takae S, Ho CH, Kawamura N, Tamura M, Hashimoto S et al. 2013 Hippo signaling disruption and Akt stimulation of ovarian follicles for infertility treatment. PNAS 110 17474-17479. (doi:10.1073/pnas.1312830110)

Kezele P \& Skinner MK 2003 Regulation of ovarian primordial follicle assembly and development by estrogen and progesterone: endocrine model of follicle assembly. Endocrinology 144 3329-3337. (doi:10.1210/en.2002-0131)

Kezele P, Nilsson E \& Skinner MK 2002 Cell-cell interactions in primordial follicle assembly and development. Frontiers in Bioscience 7 d1990-d1996. (doi:10.2741/kezele)

Li J, Kawamura K, Cheng Y, Liu S, Klein C, Liu S, Duan EK \& Hsueh AJ 2010 Activation of dormant ovarian follicles to generate mature eggs. PNAS 107 10280-10284. (doi:10.1073/pnas.1001198107)

Lima IM, Brito IR, Rodrigues GQ, Silva CM, Magalhaes-Padilha DM, Lima LF, Celestino JJ, Campello CC, Silva JR, Figueiredo JR et al. 2011 Presence of c-kit mRNA in goat ovaries and improvement of in vitro preantral follicle survival and development with kit ligand. Molecular and Cellular Endocrinology 345 38-47. (doi:10.1016/j.mce.2011.07.006)

Matos MH, Lima-Verde IB, Bruno JB, Lopes CA, Martins FS, Santos KD, Rocha RM, Silva JR, Bao SN \& Figueiredo JR 2007 a Follicle stimulating hormone and fibroblast growth factor-2 interact and promote goat primordial follicle development in vitro. Reproduction, Fertility, and Development 19 677-684. (doi:10.1071/RD07021)

Matos MH, Lima-Verde IB, Luque MC, Maia JJ, Silva JR, Celestino JJ, Martins FS, Bao SN, Lucci CM \& Figueiredo JR 2007 b Essential role of follicle stimulating hormone in the maintenance of caprine preantral follicle viability in vitro. Zygote 15 173-182. (doi:10.1017/S096719 9407004169)

Matos MH, van den Hurk R, Lima-Verde IB, Luque MC, Santos KD, Martins FS, Bao SN, Lucci CM \& Figueiredo JR 2007 c Effects of fibroblast growth factor-2 on the in vitro culture of caprine preantral follicles. Cells, Tissues, Organs 186 112-120. (doi:10.1159/000103016)

Moniruzzaman M \& Miyano T 2007 KIT-KIT ligand in the growth of porcine oocytes in primordial follicles. Journal of Reproduction and Development 53 1273-1281. (doi:10.1262/jrd.19107)

Muruvi W, Picton HM, Rodway RG \& Joyce IM 2005 In vitro growth of oocytes from primordial follicles isolated from frozen-thawed lamb ovaries. Theriogenology 64 1357-1370. (doi:10.1016/j.theriogenology. 2005.02.010)

Nilsson EE \& Skinner MK 2004 Kit ligand and basic fibroblast growth factor interactions in the induction of ovarian primordial to primary follicle transition. Molecular and Cellular Endocrinology 214 19-25. (doi:10.1016/j.mce.2003.12.001)

Nilsson E, Parrott JA \& Skinner MK 2001 Basic fibroblast growth factor induces primordial follicle development and initiates folliculogenesis. Molecular and Cellular Endocrinology 175 123-130. (doi:10.1016/ S0303-7207(01)00391-4)

Oktay K, Briggs D \& Gosden RG 1997 Ontogeny of follicle-stimulating hormone receptor gene expression in isolated human ovarian follicles. Journal of Clinical Endocrinology and Metabolism 82 3748-3751. (doi:10.1210/jcem.82.11.4346)
Oktay K, Newton H, Mullan J \& Gosden RG 1998 Development of human primordial follicles to antral stages in SCID/hpg mice stimulated with follicle stimulating hormone. Human Reproduction 13 1133-1138. (doi:10.1093/humrep/13.5.1133)

Parrott JA \& Skinner MK 1999 Kit-ligand/stem cell factor induces primordial follicle development and initiates folliculogenesis. Endocrinology $\mathbf{1 4 0}$ 4262-4271. (doi:10.1210/endo.140.9.6994)

Peng X, Yang M, Wang L, Tong C \& Guo Z 2010 In vitro culture of sheep lamb ovarian cortical tissue in a sequential culture medium. Journal of Assisted Reproduction and Genetics 27 247-257. (doi:10.1007/s10815010-9415-6)

Picton HM 2001 Activation of follicle development: the primordial follicle. Theriogenology 55 1193-1210. (doi:10.1016/S0093-691X(01)00478-2)

Picton HM, Harris SE, Muruvi W \& Chambers EL 2008 The in vitro growth and maturation of follicles. Reproduction 136 703-715. (doi:10.1530/ REP-08-0290)

Quennell JH, Stanton JA \& Hurst PR 2004 Basic fibroblast growth factor expression in isolated small human ovarian follicles. Molecular Human Reproduction 10 623-628. (doi:10.1093/molehr/gah083)

Rice S, Ojha K \& Mason H 2008 Human ovarian biopsies as a viable source of pre-antral follicles. Human Reproduction 23 600-605. (doi:10.1093/ humrep/dem390)

Rosendahl M, Andersen MT, Ralfkiaer E, Kjeldsen L, Andersen MK \& Andersen CY 2010 Evidence of residual disease in cryopreserved ovarian cortex from female patients with leukemia. Fertility and Sterility $\mathbf{9 4}$ 2186-2190. (doi:10.1016/j.fertnstert.2009.11.032)

Silva JR, van den Hurk R, de Matos MH, Dos SR, Pessoa C, de Moraes MO \& de Figueiredo JR 2004 Influences of FSH and EGF on primordial follicles during in vitro culture of caprine ovarian cortical tissue. Theriogenology 61 1691-1704. (doi:10.1016/j.theriogenology.2003.09.014)

Skinner MK 2005 Regulation of primordial follicle assembly and development. Human Reproduction Update 11 461-471. (doi:10. 1093/humupd/dmi020)

Telfer EE, McLaughlin M, Ding C \& Thong KJ 2008 A two-step serum-free culture system supports development of human oocytes from primordial follicles in the presence of activin. Human Reproduction 23 1151-1158. (doi:10.1093/humrep/den070)

Wang TR, Yan LY, Yan J, Lu CL, Xia X, Yin TL, Zhu XH, Gao JM, Ding T, Hu WH et al. 2014 Basic fibroblast growth factor promotes the development of human ovarian early follicles during growth in vitro. Human Reproduction 29 568-576. (doi:10.1093/humrep/det465)

Xu M, Barrett SL, West-Farrell E, Kondapalli LA, Kiesewetter SE, Shea LD \& Woodruff TK 2009 In vitro grown human ovarian follicles from cancer patients support oocyte growth. Human Reproduction 24 2531-2540. (doi:10.1093/humrep/dep228)

Xu J, Lawson MS, Yeoman RR, Molskness TA, Ting AY, Stouffer RL \& Zelinski MB 2013 Fibrin promotes development and function of macaque primary follicles during encapsulated three-dimensional culture. Human Reproduction 28 2187-2200. (doi:10.1093/humrep/ $\operatorname{det} 093)$

Yeh J \& Osathanondh R 1993 Expression of messenger ribonucleic acids encoding for basic fibroblast growth factor (FGF) and alternatively spliced FGF receptor in human fetal ovary and uterus. Journal of Clinical Endocrinology and Metabolism 77 1367-1371. (doi:10.1210/jcem.77.5. 8077334)

Received 21 October 2014

First decision 10 November 2014

Revised manuscript received 5 January 2015

Accepted 16 February 2015 